IRSTI 29.27.47; 29.27.17

\author{
${ }^{1}$ K.N. Dzhumagulova, ${ }^{1}$ T.S. Ramazanov, ${ }^{1}$ R.U. Masheyeva, ${ }^{2}$ Z. Donkó \\ ${ }^{1}$ Al-Farabi Kazakh National University, Almaty, Kazakhstan \\ ${ }^{2}$ Institute for Solid State Physics and Optics, Wigner Research Centre \\ of the Hungarian Academy of Sciences, Budapest, Hungary \\ "e-mail: masheyeva.ranna@gmail.com
}

\title{
Influence of background gas and external magnetic field on the localization of particles in two dimensional yukawa systems
}

\begin{abstract}
We investigate the simultaneous effect of a static homogeneous external magnetic field and a background gas medium on the quasi-localization of the dust particles - characterized quantitatively by cage correlation functions - in strongly coupled two-dimensional Yukawa systems. We apply the Langevin dynamics computer simulation method in which the frictional and Lorentz forces are taken into account. Both the presence of the magnetic field and the friction originating from the background gas, when acting alone, increase the caging time. When present simultaneously, however, we find that their effects combine in a non-trivial manner and act against each other within a window of the parameter values. For a fixed magnetic field, the increasing friction was found to first decrease the caging time and then to increase it beyond a certain value of the friction coefficient that depends on the magnetic field strength. A qualitative explanation was given for these observations based on the analysis of the peculiarities of the trajectories of individual particles.
\end{abstract}

Key words: cage correlation, dusty plasma, Langevin dynamics simulation.

\section{Introduction}

Strongly coupled plasmas are characterized by a pair-interaction potential energy that dominates the average kinetic energy of the particles [1]. Systems with this property can be described by the "onecomponent plasma" (OCP) model, which considers explicitly only a single type of charged species and assumes an inter-particle potential that accounts for the presence and effects of the other type(s) of species. The polarizable form of the interaction potential is the Yukawa type, while the nonpolarizable form is the Coulomb type; the corresponding systems are, respectively, quoted as Coulomb-OCP and Yukawa-OCP (YOCP). This latter type represents an important model system for dusty plasmas, for example, refs. [2-5]. In the simulations of these systems, the background plasma environment is accounted for by the screening of the Coulomb potential. The presence of the gaseous environment may be taken into account by using the Langevin simulation approach [6-9], where two additional terms are incorporated into the equation of motion. One of the terms represents a friction by a homogeneous background, while the other term adds momentum to the particles in form of random kicks that change the direction of the motion. The action of these two terms can be balanced to reach a desired system temperature.

The possibility of the presence of an external magnetic field has opened a new area of dusty plasma research. Interesting new effects have been observed in the experiments [10-12], which, however, are closely related to the influence of the magnetic field on the discharge plasma that embeds the dust suspension itself. The reason for this is that due to the specific charge-to-mass ratios, the electrons and ions become very strongly magnetized before any effect on the dust particles sets on. Understanding the effects observed experimentally 
is difficult because of the very demanding computational needs-simulation of dusty plasma experiments with strong magnetic fields is certainly an emerging area [13]. To overcome the problem of the magnetization of the discharge plasma, another approach, based on the equivalence of the (magnetic) Lorentz force and the Coriolis force experienced in a rotating frame of reference, was advised in Refs. [14, 15]. Implementation of this method and a successful identification of magnetoplasmons in a "quasi-magnetized" rotating dusty plasma were reported by Hartmann et al. [16].

Most of the computational studies have, meanwhile, concentrated on idealized systems, where the dust particles experience the effect of the magnetic field, whereas the surrounding medium is left unperturbed by this field. Many of the properties of such idealized systems have been studied by many-body (typically Molecular Dynamics) simulations. Collective excitations have been explored in Refs. [17, 18]; the effect of the magnetic field on the diffusion was analysed in Refs. [19, 20], while heat transport was addressed by Ott et al. [21].

Many of the properties of the strongly coupled complex plasmas are strongly related to one of its outstanding features, the quasi-localization of the particles in these systems [22]. A mathematical framework based on tracking the surroundings of individual particles has been developed by Rabani et al.[23]. The duration of the localization can be quantified by means of the so-called "cage correlation functions". The effect of a static uniform external magnetic field on the cage correlation functions in frictionless two-dimensional Yukawa systems has been investigated by Dzhumagulova et al., [24] while the effect of the friction force, induced by the presence of the buffer gas, has been addressed by Dzhumagulova et al. [25] Here, our aim is to study the simultaneous effect of the magnetic field and the friction on the cage correlation functions. The interplay of these two effects is an open question that can only be answered by a systematic parametric study due to the inherent non-linearity of the system under investigation. Our studies are based on Langevin dynamics (LD) simulation into which a proper description of the movement of the particles under the influence of an external magnetic field is incorporated [26-28].

Our numerical integration scheme of the particles' equations of motion follows the approach of Ref. [29], which takes into account the external magnetic field in the expansion of positions and velocities in the Taylor series. In Ref. [30], we introduced the friction force into the Velocity Verlet scheme, which is used in the present simulations. The scheme has been verified via comparisons of the cage correlation functions obtained in the limiting cases, when the friction force or the Lorentz force tends to be zero.

The model and the computational methods are described in Section 2, while the results are presented in Section 3. A brief summary is given in Section 4.

\section{Model and Method}

We apply the following form for the interaction potential of the particles, which had a screening property of the surrounding plasma environment:

$$
\phi(r)=\frac{Q}{4 \pi \varepsilon_{0}} \frac{\exp \left(-r / \lambda_{D}\right)}{r},
$$

where $Q$ is the charge of the particles and $\lambda_{D}$ is the screening (Debye) length.

We study a two-dimensional (2D) system; the particles move in the $(x, y)$ plane, and the magnetic field is assumed to be homogeneous and directed perpendicularly to the layer of the particles, that is $\mathbf{B}=(0,0, \mathbf{B})$. The equation of motion of the particles (given here for particle $i$ ) is:

$$
m \ddot{\mathbf{r}_{i}}(t)=\sum_{i \neq j} \mathbf{F}_{i j}\left(r_{i j}\right)+Q\left[\mathbf{v}_{i} \times \mathbf{B}\right]-v m \mathbf{v}_{i}(t)+\mathbf{F}_{B r},
$$

where the first term on the right side gives the sum of inter-particle interaction forces (to be computed for $(i, j)$ particle pairs that are separated by a distance $r_{i j}$ ), the second is the Lorentz force, and the third term represents the friction force (proportional to the particle velocity, $v$ is the friction coefficient of the dust particles in the background gaseous environment), while the fourth term represents an additional randomly fluctuating "Brownian" force.

The ratio of the inter-particle potential energy to the thermal energy is expressed by the coupling parameter

$$
\Gamma=\frac{Q^{2}}{4 \pi \varepsilon_{0} a k_{B} T},
$$


where $T$ is temperature, and $a=(1 / \pi n)^{-1 / 2}$ is the 2D Wigner-Seitz radius, with $\mathrm{n}$ being the areal number density of the particles. We introduce the screening parameter $\kappa=a / \lambda_{D}$. The strength of the magnetic field is expressed in terms of

$$
\beta=\frac{\Omega}{\omega_{p}}
$$

where $\omega_{p}=\sqrt{n Q^{2} / 2 \varepsilon_{0} m a}$ is the nominal $2 \mathrm{D}$ plasma frequency, and $\Omega=Q B / m$ is the cyclotron frequency. The strength of the friction is defined by the dimensionless parameter

$$
\theta=\frac{v}{\omega_{p}}
$$

So, the system is fully characterized by four parameters: $\Gamma, \kappa, \beta$ and $\theta$.

We apply Langevin Dynamics (LD) simulation method to describe the motion of the particles governed by the equation of motion (2). To integrate this equation, a new numerical scheme based on the Taylor expansion of the particle acceleration and velocity, followed by the correct choice of all the terms that are not higher than $O\left((\Delta t)^{2}\right)$ is used. The scheme was obtained by applying the technique developed by Spreiter and Walter, [29] but takes into account the friction force [30].

We use the localization of the particles characterized by the cage correlation function by using the method of Ref. [23], which allows the tracking of the changes in the surroundings of individual particles. We use a generalized neighbor list $l_{i}$ for particle $i, l_{i}=\left\{f\left(r_{i, 1}\right), f\left(r_{i, 2}\right) \ldots f\left(r_{i, N}\right)\right\}($ $f_{i, i}$ is excluded from the neighbour list, i.e. only "real" neighbours are taken into account). Due to the underlying sixfold symmetry of the system, we always search for the six closest neighbours of the particles, and the $f-s$ corresponding to these particles are set to a value 1 , while all other $f-s$ are set to 0 . The similarity between the surroundings of the particles $t=0$ and $t>0$ is measured by the list correlation function (defined as the normalized scalar product of the list correlation functions at time stand 0 ):

$$
C_{l}(t)=\frac{\left\langle l_{i}(t) l_{i}(0)\right\rangle}{\left\langle l_{i}(0)^{2}\right\rangle}
$$

where $\langle\ldots\rangle$ denotes averaging over particles and initial times. Obviously, $C_{l}(t=0)=1$, and $C_{l}(t)$ is a monotonically decaying function.

The number of particles that have left the original cage of particle $i$ at time $t$ can be determined as

$$
N_{i}^{\text {out }}(t)=\left|\ell_{i}(0)^{2}\right|-\ell_{i}(0) \cdot \ell_{i}(t)
$$

here, the first term gives the number of particles around particle $i$ at $t=0$, which equals to six in our case. The second term gives the number of "original" particles that remained in the surrounding after time $t$. The cage correlation function $C_{\text {cage }}$ is obtained by averaging the function $\Theta\left(c-n_{i}^{\text {out }}\right)$ over particles and initial times, that is,

$$
C_{\text {cage }}^{c}(t)=\left\langle\Theta\left[c-n_{i}^{\text {out }}(0, t)\right]\right\rangle .
$$

where $\Theta$ is the Heaviside function. We compute the cage correlation functions for, $c=3$ and take the definition of the "caging time" introduced by Donkó et al., [31] according to which $t_{\text {cage }}$ is defined as the time when $C_{\text {cage }}^{3}(t)$ decays to a value 0.1 .

The number of simulated particles is fixed at $N=1000$ that move within a quadratic simulation box. The positions of the particles are chosen randomly at the initialization of the simulations, and their velocities are sampled from a Maxwell distribution with a temperature that corresponds to the value of specified $\Gamma$. During the initial phase of the simulations, the system is thermalized, but thermostation is stopped before the data collection phase starts.

\section{Results}

Below we present the results of our simulations obtained for the cage correlation functions under the conditions of the simultaneous presence of the external magnetic field and the friction. In order to use the same numerical scheme throughout our 
work, we had to use finite values of the magnetic field and the friction coefficient. The field-free and/or frictionless cases are approximated by using very small values of these coefficients $\left(10^{-6}-10^{-5}\right)$ in the simulations. The results obtained this way approximate the "true" $\beta=0$ and/or $\theta=0$ results well within the statistical noise of the results. Nonetheless, at the presentation of the results, we give the precise (low) values of these coefficients used in the respective simulations.

Figure 1a shows the $C_{\text {cage }}^{3}(t)$ functions obtained at fixed system parameters $k=2$ and $\Gamma=20$ at a magnetic field $\beta=0.5$, with the friction coefficient $\theta$ scanned over the domain between $10^{-5}$ (representing a case with vanishing friction) and 0.5 (representing a case with high friction). In panel (b) of the same figure, another set of data is presented for a stronger magnetic field of $\beta=1.0$. One plasma oscillation period, disregarding the effect of the magnetic field, corresponds to $\omega_{E} T \sim 2 \pi$, where $\omega_{E}$ is the Einstein frequency [32] that reflects the slowing down of the dynamics due to the effect of the screening. At the given value of the screening parameter $k=2$ we find
$\omega_{E} / \omega_{p} \cong 0.49$

[32],

resulting

in $\omega_{p} T \approx 4 \pi \approx 12.6$. As a general observation, we can note that the cage correlation functions decay to the 0.1 value - specified to correspond to the caging time - on the time scale of $2-5$ plasma oscillations for the conditions of Figure 1. Such a long decay is characteristic for strong-coupled plasmas where the time scale for the diffusion of the particles is slower compared to the plasma oscillations, i.e. the particles are "quasi-localized" on the potential surface [22].

We can also observe, by comparing panels (a) and (b) of Figure 1, that the increasing friction has a more significant effect on the correlation function in the lower $-\beta$ case. At $\beta=0.5$, the correlation function increases monotonically with increasing $\theta$, however, for $\beta=1.0$ a closer observation of the behavior (see the inset in Figure 1 b)) reveals a nonmonotonic behavior. The increasing $\theta$ shifts the crossing of the correlation functions with the $C_{\text {cage }}=0.1$ line towards lower times first, and beyond $\theta \approx 0.1$ this trend reverses and remains the same for higher friction values. (a)

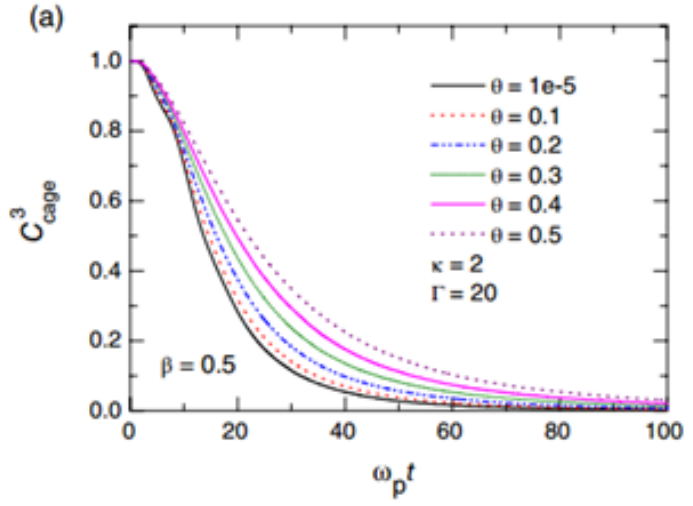

(b)

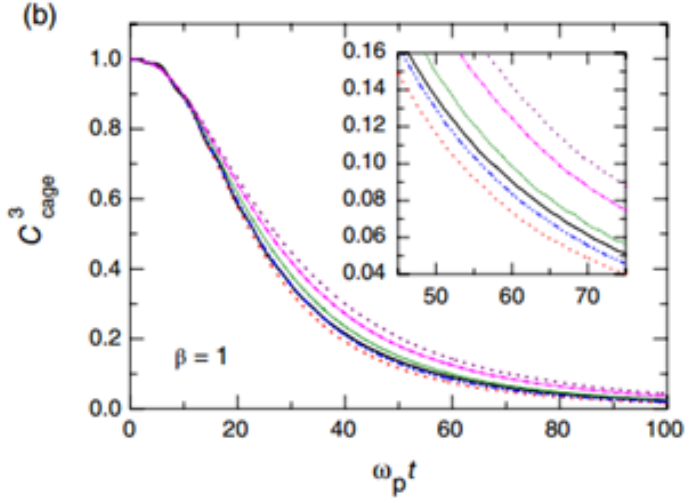

Figure 1 - Cage correlation functions for $\Gamma=20$ and $k=2$ for (a) $\beta=0,5$ and (b) $\beta=1,0$ for a wide range of the friction coefficient $\theta$. The legend shown in (a) also holds for panel (b). The inset in (b) zooms at the region when the correlation functions cross the $C_{\text {cage }}=0.1$ line (at times that correspond to the caging time)

The effect of a changing strength of magnetic field on the cage correlation functions is presented in Figure 2 for $\Gamma=20$ and $\kappa=2$, for the $\theta=0.1$ (panel (a)) and $\theta=0.5$ values of the friction coefficient. The correlation functions increase monotonically with increasing $\beta$ in both cases, a stronger influence is found at the lower value of friction (panel (a)).
The non-monotonic dependence of the caging time on the friction coefficient is further analysed in Figure 3. At small values of the magnetic field, the caging time increases monotonically with increasing friction. At $\beta>0,5$, however, as already indicated in Figure 1b, this dependence is non-monotonic. The effect - that the caging time first decreases as a function of $\theta$-becomes more pronounced at higher 
magnetic fields. The value of $\theta$ where the minimum of $T_{\text {cage }}$ occurs $\left(\theta_{\text {min }}\right)$, as well as the $\theta$ value $\left(\theta_{\text {cross }}\right)$ where the effect of magnetic field and friction "compensate each other", that is, when $T_{\text {cage }}$ becomes the same again as at $\beta=0$, both increase with increasing magnetic field.
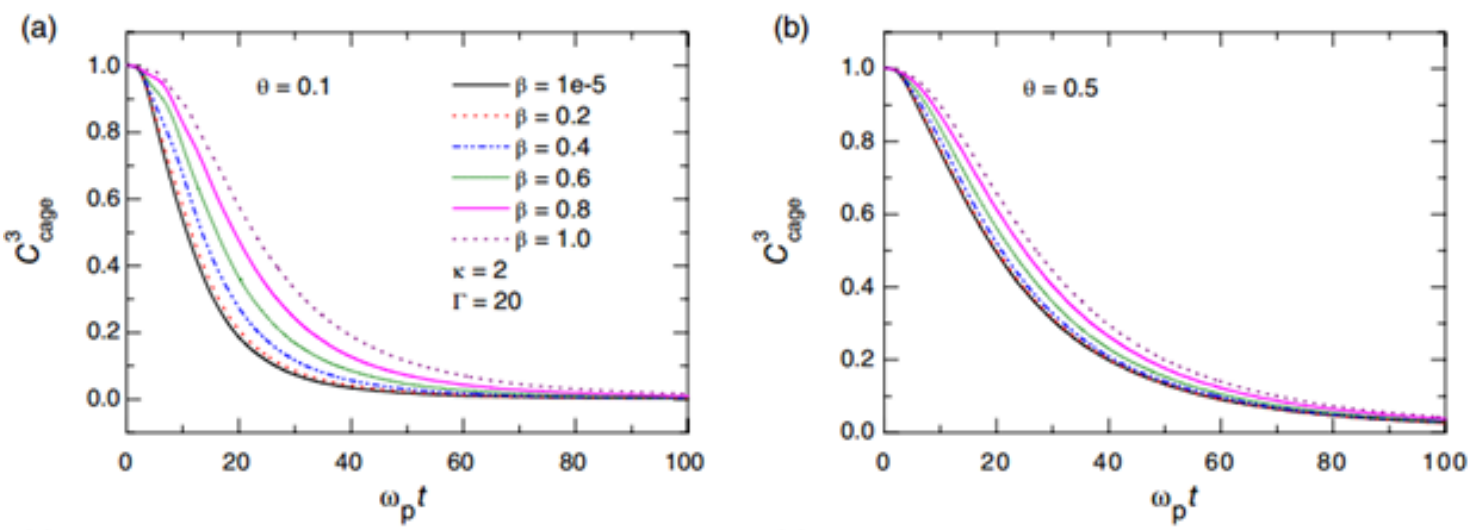

Figure 2 - Cage correlation functions for $\Gamma=20$ and $k=2$ for (a) $\theta=0.5$ and (b) $\theta=1.0$ for a wide range of the reduced magnetic field $\beta$

The interplay of the magnetic field and the friction is non-trivial. Both mechanisms, when acting alone, are known to increase the caging time. The magnetic field results in this by forcing the particles to move on circular trajectories. When the Larmor radius is smaller than the interparticle separation, diffusive motion across the field lines is significantly hindered, and the caging time is enlarged [24]. The effect of friction on the caging time is similar [25] but results from a different physical mechanism. As explained earlier, the presence of the gaseous environment is modelled via a damping mechanism by the background (as a continuum) and by a random ("Brownian") force that emulates random kicks by gas particles. The first of these slows down the particle motion, while the second increases the energy of the particle but randomizes its direction of velocity. The inverse of the frequency (related to the friction coefficient as $\theta=v / \omega_{p}$ ) can be viewed as the timescale for the change of the direction of velocity.

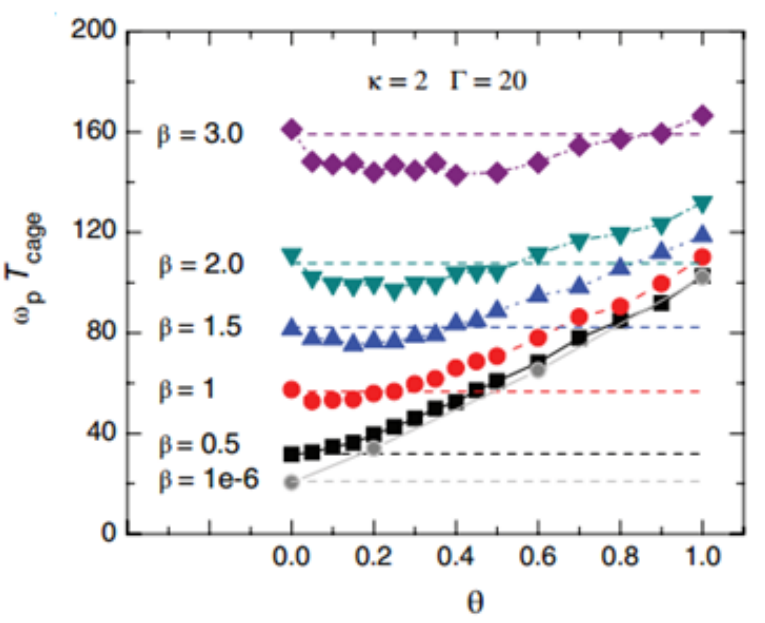

Figure 3 - Dependence of the caging time on the friction parameter $\theta$ at given values of $\beta$ in the highly magnetized domain. Note the non-monotonic dependence of $T_{\text {cage }}$ on $\theta$ for the $\beta>0$ cases.

The dashed horizontal lines correspond to $T_{\text {cage }}$ at $\theta=0$ 


\section{Conclusion}

In this paper, we have investigated the simultaneous effect of friction induced by the gas environment, as well as a homogeneous external magnetic field, on the quasi-localization of dust particles in a 2D layer. The system has been described by LD simulation. We have found that, when acting alone, both an increasing friction coefficient and an increasing strength of the magnetic field enhance the caging of the particles, as quantified by the cage correlation functions. When present simultaneously, however, a nontrivial interplay of the two effects was observed. For a fixed magnetic field $(\beta>0)$, the increasing friction was found to first decrease the caging time and then to increase it beyond a certain value of the friction coefficient that depends on the magnetic field strength. A qualitative explanation was given for these observations based on the analysis of the peculiarities of the trajectories of individual particles; however, a more detailed, quantitative understanding of the effect calls for further studies that include the analysis of the velocity autocorrelation function of the particles.

\section{Acknowledgment}

The authors acknowledge support within the Grant AP05132665 of the Ministry of Education and Science of the Republic of Kazakhstan.

1. Books (Edited Volume or Chapter Within an Edited Volume): Editor's Last name, First name, ed. Title. City: Publisher, Year.

\section{References}

1. Kalman G. J., Blagoev K., Rommel M. E., ed. Strongly Coupled Coulomb Systems. City: New York, Plenum Press, 1998.

2. Mendis D. A., Rosenberg M. "Cosmic Dusty Plasma." Annu. Rev. Astron. Astrophys. 32. (1994): 419-463.

3. Bonitz M., Henning C., Block D. "Complex plasmas: a laboratory for strong correlations." Rep. Prog. Phys. 73. (2010): 066501.

4. Thomas H., Morfill G. E., Demmel V., Goree J., Feuerbacher B., Möhlmann D. "Plasma Crystal: Coulomb Crystallization in a Dusty Plasma." Phys. Rev. Lett. 73. (1994): 652.
5. Chu J. H., I. Lin. "Direct observation of Coulomb crystals and liquids in strongly coupled rf dusty plasmas." Phys. Rev. Lett. 72. (1994): 724009.

6. Ramazanov T. S., Dzhumagulova K. N. "Shear viscosity of dusty plasma obtained on the basis of the langevin dynamics." Contrib. Plasma Phys. 48. (2008): 357.

7. Vaulina O. S., Adamovich X. G., Petrov O. F., Fortov V. E. "Evolution of the mass-transfer processes in nonideal dissipative systems. I. Numerical simulation." Phys. Rev. E. 77. (2008): 066403.

8. Liu B., Goree J. "Simulation of ThreeDimensional Dusty Plasmas." IEEE Trans. Plasma Sci. 42. (2014): 2686.

9. Arp O., Caliebe D., Piel A. "Cavity dynamics and particle alignment in the wake of a supersonic projectile penetrating a dusty plasma." Phys. Rev. E. 83. (2011): 066404.

10. Samsonov D., Zhdanov S., Morfill G., Steinberg V. "Levitation and agglomeration of magnetic grains in a complex (dusty) plasma with magnetic field." New J. Phys. 5. (2003): 24.

11. Reichstein T., Wilms J., Greiner F., Piel A., Melzer A. "Experiments and Simulations of Particle Flows in a Magnetized Dust Torus." Contrib. Plasma Phys. 52. (2012): 813.

12. Thomas Jr. E., Lynch B., Konopka U., Merlino R. L., Rosenberg M. "Quasi-discrete particle motion in an externally imposed, ordered structure in a dusty plasma at high magnetic field." Phys. Plasmas. 22. (2015): 030701.

13. Hartmann P. "Imposed ordered structure in magnetized discharge." 70 th Annual Gaseous Electronics Conf., Pittsburgh, PA, 2017.

14. Kählert H., Carstensen J., Bonitz M., Löwen H., Greiner F., Piel A. "Magnetizing a Complex Plasma without a Magnetic Field." Phys. Rev. Lett. 109. (2012): 155003.

15. Bonitz M., Kählert H., Ott T., Löwen H. "Magnetized strongly coupled plasmas and how to realize them in a dusty plasma setup." Plasma Sources Sci. Technol. 22. (2013): 015007.

16. Hartmann P., Donkó Z., Ott T., Kählert H., Bonitz M. "Magnetoplasmons in Rotating Dusty Plasmas." Phys. Rev. Lett. 111. (2013): 155002.

17. Bonitz M., Donkó Z., Ott T., Kählert H., Hartmann P. "Nonlinear magnetoplasmons in strongly coupled Yukawa plasmas."Phys. Rev. Lett. 105. (2010): 55002. 
18. Hou L.J., Shukla P. K., Piel A., Miškovic Z. L. "Wave spectra of two-dimensional Yukawa solids and liquids in the presence of a magnetic field." Phys. Plasmas. 16. (2009): 073704.

19. Feng Y., Goree J., Liu B., Intrator T. P., Murillo M. S. "Superdiffusion of two-dimensional Yukawa liquids due to a perpendicular magnetic field." Phys. Rev. E. 90. (2014): 013105.

20. Ott T., Bonitz M. "Diffusion in a Strongly Coupled Magnetized Plasma." Phys. Rev. Lett. 107. (2011): 135003.

21. Ott T., Bonitz M., Donkó Z. "Effect of correlations on heat transport in a magnetized strongly coupled plasma.” Phys.Rev.E. 92. (2015): 063105.

22. Golden K. I., Kalman G. J. "Quasilocalized charge approximation in strongly coupled plasma physics." Phys. Plasmas. 7. (2000): 14-32.
23. Rabani E., Gezelter J. D., Berne B. J. "Calculating the hopping rate for self-diffusion on rough potential energy surfaces: Cage correlations." J. Chem. Phys. 107. (1997): 6867-6876.

24. Dzhumagulova K. N., Masheeva R. U., Ramazanov T. S., Donkó Z., Dzhumagulova K. N., Masheeva R. U., Ramazanov T. S., Donkó Z. "Simultaneous effect of an external magnetic field and gas-induced friction on the caging of particles in two-dimensional Yukawa systems." Phys. Rev. E. 89. (2014): 033104.

25. Dzhumagulova K. N., Masheyeva R. U., Ramazanov T. S., Donkó Z. "Effect of Buffer Gas Induced Friction on the Caging of Particles in a Dusty Plasma." Contrib. Plasma Phys. 562. (2016): 215-220. 